\title{
Model-Based Closed-Loop Control of the Hydraulic Fracturing Process
}

\section{Authors: Qiuying Gu \& Karlene A. Hoo}

This document is the unedited author's version of a Submitted Work that was subsequently accepted for publication in Industrial \& Engineering Chemistry Research, copyright $\odot$ American Chemical Society after peer review. To access the final edited and published work, see https://dx.doi.org/10.1021/ie5024782.

Gu, Qiuying, and Karlene A. Hoo. "Model-Based Closed-Loop Control of the Hydraulic Fracturing Process." Industrial \& Engineering Chemistry Research 54, no. 5 (February 2015): 1585-1594. DOI: https://dx.doi.org/10.1021/ie5024782.

Made available through Montana State University's ScholarWorks scholarworks.montana.edu 


\title{
Model-Based Closed-Loop Control of the Hydraulic Fracturing Process
}

\author{
Qiuying $\mathrm{Gu}^{\dagger}$ and Karlene A. Hoo*,* \\ ${ }^{\dagger}$ Department of Chemical Engineering, Texas Tech University, Lubbock, Texas 79409-3121, United States \\ ${ }^{\ddagger}$ Department of Chemical \& Biological Engineering, Montana State University, Bozeman, Montana 59717-2580, United States
}

\begin{abstract}
:
Hydraulic fracturing is a technique for enhancing the extraction of oil and gas from deep underground sources. Two important goals during this process are to achieve a final fracture with a predefined geometry and to have a proper distribution of proppant material within the fracture to keep the fracture walls open and allow oil and gas to flow to the surface. The hydraulic fracturing system contains limited real-time measurements of the actual fracture conditions largely due to the remote subterranean location where the fracture propagates. The fracturing process is characterized by multiphase transport, proppant settling, and coupling of fluid and fracture growth mechanics, all occurring within a time-varying spatial domain. These features present a challenge for the implementation of online feedback control of the fracture growth and proppant placement, and there are very few accounts of attempting this goal in the open literature. To address these issues, the current work proposes a control strategy that allows for closed-loop model-based control of the hydraulic fracturing process. Previous work introduced a dynamic fracture model capable of describing the fracture propagation, fluid and particle transport, proppant bank formation, and fracture closure processes necessary to determine the fracture state evolution and predict the fracture's final performance. The QDMC (quadratic-dynamic matrix control) form of model-based control is studied. A particle filter provides a means for effective state estimation due to limited real-time measurements. Controlling the fracture geometry and proppant distribution within a hydraulic fracture is a novel application for real-time model-based control. Results of a numerical study are provided to demonstrate the performance of the closed-loop system.
\end{abstract}

\section{INTRODUCTION}

Petroleum and natural gas remain a vital part of the global energy supply. In recent years, the extraction of underground resources whose capture was previously considered to be infeasible economically has become possible due to the application of well-stimulation techniques, the foremost of which is hydraulic fracturing. ${ }^{1}$ During hydraulic fracturing, high pressure fluids are injected deep underground in order to break the rock formation and provide a highly conductive channel for resources to flow back to the wellbore.

Excellent overviews of the entire hydraulic fracturing process, with in-depth exploration of modeling and design issues, are available in the literature, $1-3$ while a brief summary of the procedure is given here. In order to create the fracture, small explosions are set off at spaced intervals at the end of the wellbore to create initial paths (perforations) into the rock formation. Next, fluid is injected, at a high pressure inside the wellbore to initiate fractures of the rock at the perforated sites. Then, a slurry consisting of fracturing fluids, additives, and proppant is pumped into the wellbore at a sufficiently high pressure and flow rate to further break the formation and extend the fractures. The addition of proppant, usually one of several special types of sand chosen for their properties, allows for the fracture walls to remain open after the fracturing fluids leak (called leak-off) into the rock. At the end of pumping, the fracture naturally closes by the surrounding rock stress, trapping the proppant. The trapped proppant provides a high conductivity, a quantity that depends on the propped fracture geometry. Achievement of a highly conductive fracture requires the placement of sufficient and uniformly distributed proppant throughout the fracture.

The current state of hydraulic fracturing is primarily an openloop process that starts with the results of a shorter and smaller scale test (called a mini-frac) in order to obtain preliminary information about the geological formation. This information is used to parametrize a mathematical model, which describes to some degree the fundamental phenomena of hydraulic fracturing, in order to design a fracturing schedule. An offline optimization then proceeds to determine flow rates and material concentrations for the entire process in advance of the main fracturing process, and online adjustments occur only in the case of large abnormalities (e.g., pressure spikes) in the measurements. Consider the potential of a closed-loop process that makes use of the measured data to update the treatment design as the fracturing process proceeds. Such an approach enables automatic manipulation of flow rates and proppant concentration that may result in improved fracture performance and reduced costs. Online data for closed-loop control may come from downhole measurements of pressure, temperature, and microseismic events, combined with predictions from a suitable mathematical model of the system. 
As a consequence of the limited availability of measurements within the hydraulic fracturing system, a key component of the model-based controller design is the online estimation of both the parameters and states from the measured process data. This may present difficulties, as the hydraulic fracturing process is described by a system of nonlinear partial differential equations. This distributed parameter system (DPS) contains spatially varying outputs, states, and parameters. An additional complication is that the solution domain varies with time as the hydraulic fracture propagates.

A well-known state estimation technique for use with nonlinear models is the extended Kalman filter (EKF) wherein a Gaussian posterior density is assumed, and a first-order Taylor series expansion provides a local approximation of the state. ${ }^{4}$ However, it is well-known that the EKF may give a high estimation error when strong nonlinearities are present. More recent alternatives for state estimation include the ensemble Kalman filter or a particle filter. The ensemble Kalman filter (EnKF) was introduced by Evensen ${ }^{5}$ and has found use in a variety of applications. ${ }^{6-9}$ The EnKF is a type of particle filter ${ }^{10}$ that relies on the key assumption that the prior probability density function (pdf) of the states of the system has a Gaussian distribution. This is in contrast to the larger class of particle filters that makes no such assumption. ${ }^{11}$ Particle filtering is a Bayesian approach to dynamic state estimation with a recursive predictor-corrector structure. ${ }^{12}$ The filter is initiated by choosing a set of state estimates or "particles" sampled from the initial distribution of the states. Then, as those sample points propagate through the nonlinear system model, their relative contribution to the probability distribution function is updated, and the best estimate of the state of the system can be obtained based on this ensemble distribution. ${ }^{10}$ In this paper, particle filtering is used for online state estimation.

In order to apply a model-based control approach to regulate a DPS, a reduced-order model (ROM) must be derived or identified, and there exists a significant body of research on obtaining such an ROM. ${ }^{13-20}$ To control a linear DPS, Balas ${ }^{21}$ demonstrated how to reduce an infinite-dimensional controller to a low-dimensional form, whereas Ray ${ }^{22}$ described a lumped approach to design feedback controllers. In the case of controlling a nonlinear DPS, Gauthier ${ }^{23}$ provided an algorithm to evaluate an $H_{\infty}$ controller design. In contrast, sliding mode control was studied by Hanczyc and Palazoglu. ${ }^{24}$ Christofides and co-workers used a combination of geometric and direct Lyapunov methods in the design of robust static feedback controllers for hyperbolic PDE systems. ${ }^{25,26}$ This work was extended to systems described by nonlinear parabolic equations. ${ }^{27}$ Each of these methods is applicable to a nonlinear model of a certain form. The hydraulic fracturing process is described by a complex system of equations including phase transition relations as well as intricate boundary conditions that preclude an analytical reduction to fit these forms.

There also exist several techniques to control a DPS that are dependent on data-based model reduction. In the work of Gay and Ray, a dynamic matrix controller solution was applied using a low-order model generated using SVD. ${ }^{13}$ The application of quadratic dynamic matrix control (QDMC) on a DPS can be found in ref 18 where a combined SVD-KL method was used to arrive at a low-order model. Chen and Hoo considered the problem of closed-loop optimization of a reservoir in order to maximize oil production. ${ }^{7,8}$ In that work, Markov chain Monte Carlo and ensemble Kalman filtering were used to estimate the states of the system and to address model parameter uncertainty. Using the updated states and parameters, data for low-order model identification were generated. The resulting model was used in a model predictive control strategy.

In the current work, the mathematical model of the hydraulic fracturing system and its numerical solution (subsequently referred to as the nonlinear system model) developed by $\mathrm{Gu}$ and $\mathrm{Hoo}^{28}$ is adapted for simulation of the fracturing process within a model-based feedback control framework. Particle filtering is chosen to propagate the state estimate, as this method allows the nonlinear system model to be used for describing the evolution of system states. At certain times, identification tests are performed upon the nonlinear system model, in order to identify a linear step response model, where the propagated state estimate is used to provide a starting point for the identification. This reduced-order model type can be used by the QDMC controller to optimize the hydraulic fracturing pumping schedule, which consists of the flow rate and proppant concentration. These variables are used to drive the fracture geometry and the settled bank height to follow the target trajectories associated with the desired final fracture condition.

The paper is organized as follows. Section 2 provides a short introduction of the mathematical model developed in $\mathrm{Gu}$ and Hoo. ${ }^{28}$ Section 3 provides a brief overview of the particle filter method. Also in this section, the QDMC controller and a proposed overall closed-loop framework to regulate hydraulic fracturing processes are introduced. In section 4, numerical studies to evaluate feasibility of the proposed closed-loop framework are presented and analyzed. Lastly, section 5 summarizes the findings of this study and proposes future work.

\section{COMPUTATIONAL MODEL}

A mathematical model was developed to describe fracture propagation, solids transport, proppant settling, and bank formation processes that occur during hydraulic fracturing. ${ }^{28}$ Here, a brief description of this model is given.

The mathematical model describes a PKN-type biwing vertical fracture that is assumed to propagate unidirectionally inside a single rock layer surrounded by homogeneous formation conditions. The injected proppant is assumed to travel at the carrier fluid's velocity in the propagation direction while simultaneously settling toward the fracture bottom due to gravitational effects. As proppant particles reach the fracture bottom, they begin to form a bank.

The governing equations are generated by applying the fundamental principles of mass and momentum conservation along with the use of linear elastic fracture mechanics. The local overall mass balance equation along the propagation direction is

$$
H \frac{\partial W}{\partial t}-\frac{h_{\mathrm{f}}}{48 \mu M_{\mathrm{c}}} \frac{\partial^{2} W^{4}}{\partial x^{2}}+h_{\mathrm{f}} U=0
$$

where $M_{c}$ is the fracture compliance, which is given by

$$
M_{\mathrm{c}}=\frac{2 h_{\mathrm{f}}\left(1-\nu^{2}\right)}{E}
$$

In the above equation, $H$ is the predefined fracture height, $W$ is the fracture width, $h_{\mathrm{f}}$ is the local height of the area free for to fluid flow (simply the difference between the fracture height and the bank height), and $U$ is the fluid leak-off per unit height 
accounting for both walls of the fracture. This leak-off per unit height can be described by ${ }^{29}$

$$
U=\frac{2 C_{1}}{\sqrt{t-\tau(x)}}
$$

where $C_{1}$ is the overall fluid leak-off coefficient, $t$ is the elapsed time since fracturing was initiated, and $\tau$ represents the initial time at which the current location inside the fracture became open.

The local growth of the bank height is a function of the settled proppant particles

$$
\frac{\mathrm{d}\left(H-h_{\mathrm{f}}\right) W}{\mathrm{~d} t}=\frac{C V_{\mathrm{s}} W}{1-\phi}
$$

where $V_{s}$ is the proppant settling velocity determined using Stokes law and $\phi$ is the proppant bank porosity.

By applying a material balance to the particle phase and discretizing using a finite-difference scheme, the following equation described proppant transport:

$$
\frac{\partial\left(W h_{\mathrm{f}} C\right)_{i, j}}{\partial t}+\frac{\partial q_{i, j} C_{i, j}}{\partial x}+\frac{\partial q_{i, j} C_{i, j}}{\partial y}=0
$$

where $C_{i, j}$ is the proppant volumetric concentric concentration in element $(i, j), q_{i, j}$ is the flow rate of element $(i, j)$, with $i$ being the index in the $x$-direction and $j$ being the index in the $y$ direction.

The boundary conditions for solving those equations include the following:

1. At the wellbore, the flow rate is specified by $q(x, t)=$ $Q^{0}(t)$, where $Q^{0}$ is the rate of slurry injection.

2. At the fracture tip, $x=L(t)$, the usual boundary condition is that the net pressure is zero, ${ }^{30,31}$ which leads to the condition $W(L(t), t)=0$, by way of the pressure width relation, $W=M_{c} P .{ }^{32}$

In addition, the initial condition is that the fracture is closed, that is $W(x, 0)=0$.

This system of nonlinear partial differential equations requires a numerical solution to determine the system evolution in time. A finite volume approach is used, with the fracture geometry represented by a two-dimensional mesh grid. In the vertical direction, the mesh is evenly distributed and fixed spatially and temporally. As the fracture propagates lengthwise, new elements are added at each time step to capture the fracture growth. The discretization of the above equations uses a backward in time and centralized in space finite volume approach, which is solved numerically to give (an approximate solution order at each time step): the fracture width (implicit solution), the local fluid velocities, proppant concentration, proppant bank height, and fracture length (explicit solution). The details of the computational solution procedure can be found in ref 28 .

\section{CLOSED-LOOP CONTROL OF THE FRACTURE PROCESSES}

The objective for closed-loop control of a hydraulic fracturing process is to obtain a prescribed final propped fracture geometry with associated productivity characteristics and to do so in the face of unmeasured process disturbances. It is assumed that during the design process that occurs prior to the fracturing operation, target trajectories of the fracture states are determined that, if achieved, will result in the desired final fracture condition after shut-in is complete. Design of the fracture and the associated initial treatment schedule can proceed through methods such as that described in ref 1 . Then, the controller objective is to force the current trajectories of the specified system states to follow the prescribed target trajectories during the fluid and slurry injection stages of the fracture treatment procedure. In this work, fracture width and length, proppant bank height, and proppant concentration are the desired states to be regulated. Pumping rate and proppant concentration of the injected slurry are the available manipulated variables.

3.1. Model-Based Control Framework. Implementing a model-based control scheme requires a suitable model for online optimization. In this case, a step-response model frequently identified in real-time as the hydraulic fracturing process progresses would be suitable for the QDMC strategy. Thus, these components can be used together to provide an overall feedback control framework for the hydraulic fracturing process.

A schematic of the proposed model-based control framework is shown in Figure 1. Within the overall control structure, there

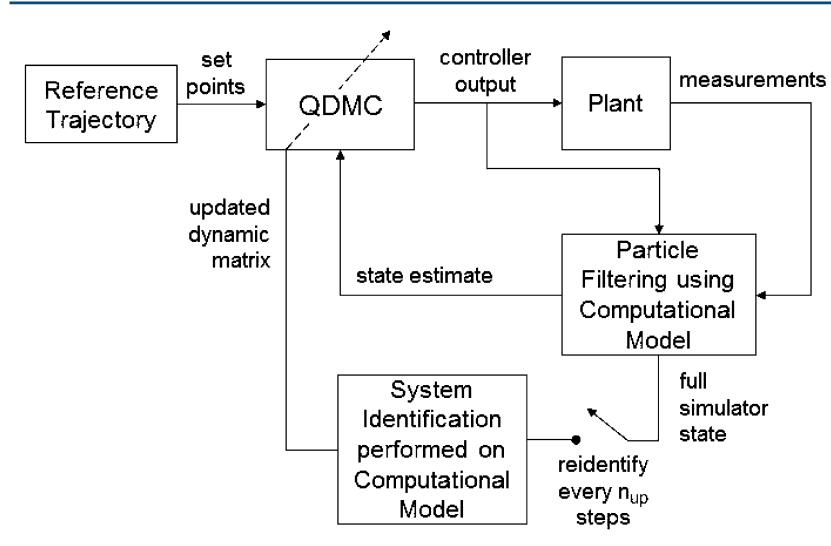

Figure 1. Proposed control framework.

exist modules for particle filtering, system identification, and QDMC control. The plant measurements and controller outputs are inputs to the particle filter, which uses the nonlinear system model to produce an ensemble state estimate to be used by the controller. This involves simulating the fracture propagation and proppant transport using the numerical solution procedure developed in ref 28 and reviewed in section 2. Periodically within the system identification module, a reidentification occurs, which involves performing a series of step test on the nonlinear system model starting from the current estimate of the plant states. The step tests are carried out by simulating the fracture using the model described in section 2, the current levels of injection rate and concentration, and either a positive or negative step change in one of these inputs. The resulting state response is sampled at the controller time step. Several different step changes in the injection rate and injection concentration are applied, followed by determination of the step response coefficients using a leastsquares approach.

This produces an updated step response model for use by the QDMC controller. The QDMC controller calculates the optimal sequence of flow rates and injected proppant concentrations, but only the first prescribed input is implemented on the process, and the computation repeats at each subsequent time step. In a related work, Chen and Hoo 
proposed a similar structure for the closed-loop optimal management of production from an oil reservoir. ${ }^{7}$

The framework introduced here combines state and parameter estimation, model reidentification, and modelbased control to provide online feedback control of the fracturing process. The following sections provide an overview of particle filtering and the quadratic dynamic matrix controller.

3.2. Particle Filtering. Currently, online measurements available for estimating subsurface behavior in the hydraulic fracturing process are limited to the downhole pressure and microseismic data. In microseismic monitoring, an array of geophones is lowered inside an observation well to record the small seismic events, such as slippage of planar rock faces that occur as the fracture propagates. ${ }^{33}$ In this work, it is assumed that the wellbore width and fracture length can be obtained from measurement of downhole pressure and microseismic data, respectively. The unmeasurable states to be regulated must be estimated; and in this work, a particle filtering approach is employed for this purpose. A short description of the use of particle filtering to estimate the unknown states and parameters of a nonlinear system follows.

A general nonlinear discrete-time system can be represented as $^{11,12,34}$

$$
\mathbf{x}_{k}=\mathbf{f}_{k}\left(\mathbf{x}_{k-1}, \mathbf{v}_{k-1} ; t\right) \quad \mathbf{x} \in \mathcal{R}^{n}, v \in \mathcal{R}^{m}
$$

where $\mathbf{x}_{k}$ is the system state vector at time $k, \mathbf{v}_{k-1}$ is the state noise vector, and $\mathbf{f}_{k}$ is a nonlinear and potentially time-varying real function describing the time evolution of the states. Information on the states $\mathbf{x}_{k}$ are only obtained through measurements, $\mathrm{z}_{k} \in \mathcal{R}^{p}$, which are related through the measurement equation:

$$
\mathbf{z}_{k}=\mathbf{h}_{k}\left(\mathbf{x}_{k}, \mathbf{n}_{k}\right) \quad \mathbf{z} \in \mathcal{R}^{p}, \mathbf{n} \in \mathcal{R}^{p}
$$

where $\mathbf{n}_{k}$ is the measurement noise vector and $\mathbf{h}_{k}$ is a potentially nonlinear and time-varying real function. It is assumed that the distributions of $\mathbf{v}$ and $\mathbf{n}$ are independent.

In the Bayesian framework of dynamic state estimation, the target is to obtain an optimal estimate of the states based on the posterior probability density function, $p\left(\mathbf{x}_{k} \mid \mathbf{z}_{1: k}\right)$, of the current system state, $\mathbf{x}_{k}$, given all of the available system information, including the vector of all measurements up to time $k, \mathbf{z}_{1: k}$. The implementation consists of two stages: prediction and update. During the prediction step, the states of each individual particle at the next time step are forecasted by the model. Once the new measurements are received at time $k$, the posterior density function of each state is updated, and an optimal estimate of the system state can be obtained based on the ensemble data.

A distinct quality of particle filtering compared to traditional observation methods such as the Kalman filter is to represent the state probability density function by a set of randomly generated samples rather than with an analytical form. The posterior density at step $k$ can be approximated as

$$
p\left(\mathbf{x}_{k} \mid \mathbf{z}_{1: k}\right) \approx \sum_{i=1}^{N_{\mathrm{p}}} \mathbf{q}_{k}^{i} \delta\left(\mathbf{x}_{k}-\mathbf{x}_{k}^{i}\right)
$$

where $N_{\mathrm{p}}$ is the number of particles, $\mathbf{x}_{k}^{i}$ is the state of particle $i$, $\mathbf{q}_{k}^{i}$ is the normalized weight assigned to particle $i$, and $\delta$ is the Dirac delta function. Because of the difficulty in sampling from the posterior density $p\left(\mathbf{x}_{k} \mid \mathbf{z}_{1: k}\right)$, samples can be drawn instead from a less complex but known distribution, $\pi\left(\mathbf{x}_{k} \mid \mathbf{z}_{1: k}\right)$. It is often the case that $\pi\left(\mathbf{x}_{k} \mid \mathbf{z}_{1: k}\right)=\pi\left(\mathbf{x}_{k} \mid \mathbf{x}_{k-1}, \mathbf{z}_{k}\right)$. This allows recursive updating of the weights according to ${ }^{12}$

$$
\mathbf{q}_{k}^{i} \propto \mathbf{q}_{k-1}^{i} \frac{p\left(\mathbf{z}_{k} \mid \mathbf{x}_{k}^{i}\right) p\left(\mathbf{x}_{k}^{i} \mid \mathbf{x}_{k-1}^{i}\right)}{\pi\left(\mathbf{x}_{k}^{i} \mid \mathbf{x}_{k-1}^{i}, \mathbf{z}_{k}\right)}
$$

One problem with this technique is the degeneracy phenomenon, wherein the particle weights for all but a single particle quickly converge to negligible values. An approach to mitigate this effect is to introduce sequential importance resampling. $^{12,35}$

The state vector, $\mathbf{x}_{k}$, to be estimated for the hydraulic fracturing process contains four components:

$$
\mathbf{x}_{k}=\left[\mathbf{W}^{\mathrm{T}} \mathbf{h}_{b}^{\mathrm{T}} \mathbf{C}^{\mathrm{T}} L\right]^{\mathrm{T}}
$$

where $L$ is the fracture length and $\mathbf{C}, \mathbf{h}_{b}$, and $\mathbf{W}$, respectively, represent vectors of concentrations, bank heights, and fracture widths at a set of selected positions along the fracture length. Alternatively, these variables could represent a vector of basis function coefficients describing the distributions of these quantities across the fracture geometry. The measurement vector contains two components and is given by

$$
\mathbf{z}_{k}=\left[W_{0} L\right]^{T}
$$

where $W_{0}$ is the width at the wellbore and $L$ is the length measurement obtained through microseismic recordings.

The state estimates (particles) are initialized by sampling from a multivariate normal distribution. During the prediction step, all states are propagated forward one time step through use of the model described in section 2 and the previous work. ${ }^{28}$ This model also provides predictions of measurements $W_{0}$ and $L$ associated with every particle. After a measurement from the plant becomes available, eq 9 is used to adjust the particle weights. The ensemble state estimate used by the controller can be obtained by using the particle with the highest weight. The particles then are resampled from an empirical cumulative distribution function formed from the updated particle weights to form a new distribution of particles. This prediction-correction-resampling procedure repeats at each sample time.

3.3. Quadratic Dynamic Matrix Control. The states to be controlled include the width, bank height, and concentration profiles and the fracture length. In section 3.1, it was proposed that a step-response model can be frequently identified from the nonlinear system model as the hydraulic fracturing process progresses, and the identified dynamic matrix can be used within a controller implementing a quadratic dynamic matrix control algorithm.

The QDMC is designed to minimize an objective function ${ }^{36}$

$$
\begin{aligned}
J= & \sum_{i=1}^{n_{\mathrm{p}}}\left(\mathbf{x}_{k+i}-\mathbf{x}_{k+i}^{*}\right)^{\mathrm{T}} Q\left(\mathbf{x}_{k+i}-\mathbf{x}_{k+i}^{*}\right) \\
& +\sum_{i=0}^{n_{\mathrm{c}}} \Delta \mathbf{u}_{k+i}^{\mathrm{T}} \mathbf{R} \Delta \mathbf{u}_{k+i}
\end{aligned}
$$

where $n_{\mathrm{p}}$ is the prediction horizon, $\mathbf{x}_{k}$ is the vector of the controlled system states at time $k+i, \mathbf{x}_{k+1}^{*}$ is the state of the reference trajectory at time $k+i, \mathbf{Q}$ is positive-definite matrix that represents the cost of the state deviations, $n_{\mathrm{c}}$ is the control horizon, $\Delta \mathbf{u}_{k+i}$ is the vector of control moves at time $k+i$, and $\mathbf{R}$ is a positive-definite matrix that penalizes excess control moves. Since the majority of the system states are unavailable in 
the fracturing process, the particle filter is used to estimate the states.

The QDMC formulation allows equality and inequality constraints on linear combinations of the system states and controller outputs. These usually take the form of upper and lower bounds on the states, controller outputs $\left(\mathbf{u}_{k+i}\right)$, and increments in the controller outputs $\left(\Delta \mathbf{u}_{k+i}\right)$. A further equality constraint on eq 12 is a step response model of the system

$$
\mathbf{x}_{k+i}=\mathbf{x}_{0}+\sum_{j=1}^{k+i} \mathbf{s}_{j} \Delta \mathbf{u}_{k+i-j}
$$

for $i=1, \ldots, n_{\mathrm{p}}, \mathbf{S}_{j}$ is the matrix of step response coefficients at lag $j$. Taking the initial state, $\mathbf{x}_{0}$, to be zero, the remaining terms can be split into two parts, as follows:

$$
\mathbf{x}_{k+i}=\sum_{j=1}^{i} \mathbf{s}_{j} \Delta \mathbf{u}_{k+i-j}+\sum_{j=i+1}^{k+i} \mathbf{s}_{j} \Delta \mathbf{u}_{k+i-j}
$$

which separates the terms that depend on controller movements before time step $k, \Delta \mathbf{u}_{k+i-j}, i<j<k+i$, and controller movements during and after time step $k, \Delta \mathbf{u}_{k+i-j}, j \leq i$. The terms depending on controller movements before time step $k$ can be combined into a single quantity, called the free response, $\mathbf{f}(k+i \mid k)$, defined as

$$
\mathbf{f}(k+i \mid k)=\sum_{j=i+1}^{k+i} \mathbf{s}_{j} \Delta \mathbf{u}_{k+i-j}
$$

Instead of using the step-response model to represent this term, an alternative is to predict this term's value through use of the nonlinear system model. This should provide a more accurate prediction. The step response model is used to predict the effects of the current and future control moves on the state trajectory, and because it has a linear form, well-known quadratic programming techniques can be used to solve the optimal control problem.

The tuning parameters for the QDMC controller design include $n_{\mathrm{p}}, n_{\mathcal{o}} \mathbf{Q}$ and $\mathbf{R}$. Equation 12 is solved subject to constraints, to determine the controller output sequence $\Delta \mathbf{u}_{k: k+n_{c}}$. Only the first move, $\Delta \mathbf{u}_{k}$ is implemented on the plant, with the entire procedure repeated at each subsequent time step.

Initial studies with the QDMC controller presented difficulties with the tuning of a large number of coefficients for the matrices $\mathbf{Q}$ and $\mathbf{R}$. Relying on the observation that the injected proppant concentration has a small effect on the fracture geometry (length and width), the fracture width and length are regulated by the pumping rate. The injected proppant concentration is used to regulate deviations in the bank height and proppant concentration trajectories.

The equation to predict the states describing fracture geometry is given by

$$
\begin{gathered}
{\left[\begin{array}{c}
\mathbf{W} \\
-- \\
L
\end{array}\right]_{k+1: k+n_{\mathrm{p}}}=\mathbf{f}_{W, L}\left(\Delta \mathbf{Q}_{0: k-1}, \Delta \mathbf{C}_{0: k-1}\right)+\mathbf{G}_{\mathbf{W}, L} \Delta} \\
\mathbf{Q}_{k: k+n_{\mathrm{c}}}
\end{gathered}
$$

where $\left[\begin{array}{ll}W^{\mathrm{T}} & L\end{array}\right]_{k+1: k+n_{\mathrm{p}}}^{\mathrm{T}}$ are width and length predictions for time steps $k$ through $k+n_{\mathrm{p}} ; \mathbf{f}_{\mathrm{W}, L}\left(\Delta \mathbf{Q}_{0: k-1}, \Delta \mathbf{C}_{0: k-1}\right)$ is the prediction of future fracture widths and lengths based upon past pumping rates $\left(\Delta \mathbf{Q}_{0: k-1}\right)$ and proppant injection concentrations $\left(\Delta \mathbf{C}_{0: k-1}\right)$; and $\mathbf{G}_{\mathbf{W}, L}$ is the dynamic matrix to predict the effects of current and future changes in the pumping rate $\left(\Delta Q_{k: k+n_{c}}\right)$ on fracture widths and lengths. Since only the first control moves are implemented this can be incorporated in the prediction of the future concentration and bank height state trajectories:

$$
\left[\begin{array}{c}
\mathbf{C} \\
-- \\
\mathbf{h}_{b}
\end{array}\right]_{k+1: k+n_{\mathrm{p}}}=\mathbf{f}_{\mathbf{C}, \mathbf{h}_{\mathrm{b}}}\left(\Delta \mathbf{Q}_{0: k}, \Delta \mathbf{C}_{0: k-1}\right)+\mathbf{G}_{\mathbf{C}, \mathbf{h}_{b}} \Delta C_{k: k+n_{\mathrm{c}}}
$$

where the left-hand side is the prediction that describes the concentration and bank height profiles within the fracture, the first term on the right-hand side predicts the effect of the past proppant concentration and the past and current pumping rate on the concentration and bank height profiles, and the last term on the right-hand side is the dynamic matrix composed of step response coefficients describing the effect of future changes of the proppant concentration on the concentration and bank height profiles.

Identification tests on the nonlinear system model show that the linear step response coefficients often were a poor representation of the true system behavior. The largest prediction errors were found to be due to the asymmetric behavior of the responses to increases versus decreases in either of the inputs. To improve the predictive ability of the linear step-response models, directional dynamic matrices $\mathbf{G}_{\mathbf{W}, L}^{+}, \mathbf{G}_{\mathbf{W}, L}^{-}$, $\mathbf{G}_{\mathbf{C}, \mathbf{h}_{b}}^{+}$, and $\mathbf{G}_{\mathbf{C}, \mathbf{h}_{b}}^{-}$were introduced. The term $\mathbf{G}_{\mathbf{W}, L}^{+}$is composed of step-response coefficients identified from only positive steps tests in $\Delta \mathbf{Q}^{0}$, while $\mathbf{G}_{\mathbf{W}, L}^{-}$is constructed with only negative step tests in $\Delta \mathbf{Q}^{0}$, and $\mathbf{G}_{\mathrm{W}, L}$ is formed from data from both types of step tests. The coefficients in the terms $\mathbf{G}_{\mathbf{C}, \mathbf{h}_{b}}^{+}, \mathbf{G}_{\mathbf{C}, \mathbf{h}_{b}}^{-}$, and $\mathbf{G}_{C, \mathbf{h}_{b}}$ are similarly obtained from step tests on $\Delta \mathrm{C}$. If the initial sequence computed by the QDMC controller using $\mathbf{G}_{\mathrm{W}, L}$ is such that the first element of $\Delta \mathbf{Q}_{k}>0$, the controller output is recomputed using $\mathbf{G}_{\mathbf{W}, L}^{+}$and vice versa. If the direction of the (1, 1) element changes after the recalculation that uses $G_{W, L}^{+}$or $\mathbf{G}_{\mathbf{W}, L}^{-}$, then the original result using $\mathbf{G}_{\mathbf{W}, L}$ is implemented on the plant; otherwise, the controller output computed using $\mathbf{G}_{\mathbf{W}, L}^{+}$or $\mathbf{G}_{\mathbf{W}, L}^{-}$is used. Analogous rules are applied for the calculation of $\Delta \mathrm{C}_{k: k+n_{c}}$.

Stability results for tuning of the QDMC controller are available from Zheng and Hoo. ${ }^{19}$ However, for the current system, the model obtained through system identification is an approximation of the actual dynamics, so the stability results of the preceding work may not be completely applicable. In section 4 the use of the QDMC controller in the proposed framework (see Figure 1) for the feedback control of the hydraulic fracturing processes is presented and analyzed.

\section{RESULTS AND DISCUSSION}

This section investigates the feasibility of the control framework proposed in Section 3, using computational studies within the MATLAB(Mathworks, Natick, MA) environment. Specifically, the ability of the closed-loop system to reject unmeasured disturbances is evaluated. The control objective is to track the selected controlled states by adjusting the pumping rate and proppant concentration. The assumption is that through accurate tracking of the target trajectories, the desired final condition of the propped fracture will be obtained. The 
hydraulic fracturing model as described in section 2 is used to represent the plant; noise will be added to both the system inputs and measurements. As noted in section 3, this model also is used by the particle filter for state estimation and by the QDMC controller in the form of a step-response model (see Figure 1).

The selected states to be controlled are (1) the fracture width and proppant bank height at 10 positions along the fracture length, (2) proppant concentration at the same 10 selected positions contained within a band located at a height of five meters above the fracture bottom, and (3) the fracture length. This gives a total of 31 states to be controlled. Previously, ${ }^{28}$ the sensitivity of the final fracture condition to pumping rates and injected proppant concentrations was studied by executing a series of different treatment designs on the model. Based on the results of that work, the experimental conditions consisting of a $0.027 \mathrm{~m}^{3} / \mathrm{s}$ injection rate and a proppant concentration of $0.27(\mathrm{v} / \mathrm{v})$ are chosen as the inputs of the model to generate the target trajectories.

The properties of the system, including formation and material parameters are provided in Table 1 . The controller

Table 1. Model Parameters and Treatment Schedule Used in the Case Study,30

\begin{tabular}{lcl}
\multicolumn{1}{c}{ definition } & symbol & \multicolumn{1}{c}{ value } \\
proppant diameter & $d$ & $1000 \mu \mathrm{m}$ \\
fluid-loss coefficient & $C_{\mathrm{l}}$ & $6.3 \times 10^{-5} \mathrm{~m} / \sqrt{\mathrm{s}}$ \\
Young's modulus of the formation & $E$ & $10^{4} \mathrm{MPa}$ \\
proppant permeability & $K_{\mathrm{f}}$ & $500 \mathrm{Darcy}$ \\
rock permeability & $K_{\mathrm{r}}$ & $0.01 \mathrm{mD}$ \\
vertical fracture height & $\mathrm{H}$ & $10 \mathrm{~m}$ \\
proppant particle density & $\rho_{\mathrm{sd}}$ & $2648 \mathrm{~kg} / \mathrm{m}^{3}$ \\
pure fluid density & $\rho_{\mathrm{f}}$ & $1000 \mathrm{~kg} / \mathrm{m}^{3}$ \\
pure fracturing fluid viscosity & $\mu$ & $0.56 \mathrm{~Pa} \cdot \mathrm{s}$ \\
Poisson ratio of the formation & $\nu$ & 0.2 \\
proppant bank porosity & $\phi$ & 0.12
\end{tabular}

time step and measurement sampling time are both set at $25 \mathrm{~s}$. The initial pumping rate is set as $0.027 \mathrm{~m}^{3} / \mathrm{s}$ for 20 sampling steps. The particle filter was initialized at time 0 , while feedback control was initialized at time step $20(t=500 \mathrm{~s})$. A decrease (step change) by $1 / 2$ of the normal pumping rate $\left(0.014 \mathrm{~m}^{3} / \mathrm{s}\right)$ occurred at time step 20. In the case of no control, the injection rate was unchanged from $0.027 \mathrm{~m}^{3} / \mathrm{s}$, thus the effective flow into the fracture was $0.013 \mathrm{~m}^{3} / \mathrm{s}$.

Proppant injection was scheduled to occur at time step 80 $(2000 \mathrm{~s})$. In the case of feedback control, the controller determined the injected proppant concentration from $2000 \mathrm{~s}$ onward. In contrast, without feedback control, the injected proppant concentration remained at the designed condition of 0.27 (v/v). Pumping continued until time step 106 for a total treatment time of $2650 \mathrm{~s}$. Fracture shut-in occurred until fluid leak-off to the formation was complete. This represented approximately $3600 \mathrm{~s}$.

The QDMC control was implemented with the flow rate manipulated to control the overall fracture geometry (width and length) while the proppant injection concentration was used to regulate both the suspended and settled proppant distributions (concentration and bank height). The prediction and control horizons (not optimized) were chosen to be 15 time steps (375 s) and 5 time steps (125 s), respectively. The selected weights for each of the controlled variables in the matrix $Q$ approximately correspond to an inverse of the order of magnitude of each variable value (effectively providing a scaling on the errors in these states). The values of the weights chosen are $Q_{L}=1 \times 10^{-6}, Q_{W}=1, Q_{C}=1 \times 10^{-2}$, and $Q_{h}=1 \times$ $10^{-4}$.

The penalty for control moves for each variable were given a value of $R_{Q 0}=R_{C 0}=10$. Reidentification of the step response model was performed every five sampling times. Combined with the prediction horizon (15), this meant that the step response data had to be obtained for 20 time steps during every reidentification. For state estimation, the ensemble consisted of 20 particles, with resampling occurring at every time step.

Figure 2 shows the trajectory of the pumping rate for the case of feedback control. Once the disturbance of inlet flow rate

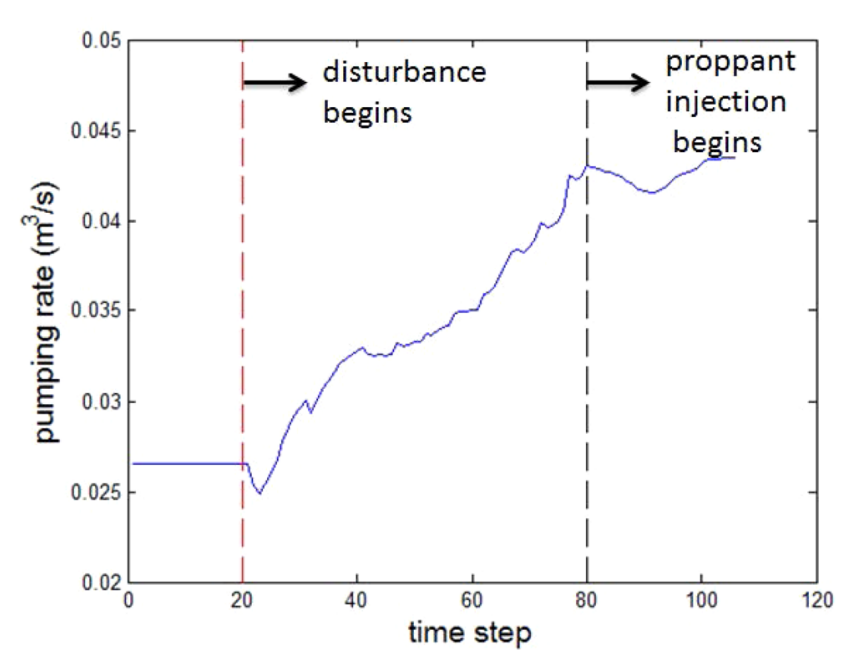

Figure 2. Variations in manipulated pumping rate in the case of feedback control.

begins at time step 20, the controller reacts by increasing the pumping rate. Figure 3 shows the resulting fracture length changes. The adjustment of flow rate by the feedback controller brings the length closer to the desired trajectory compared to the case with no control. Clearly some deviation still persists even with the controller.

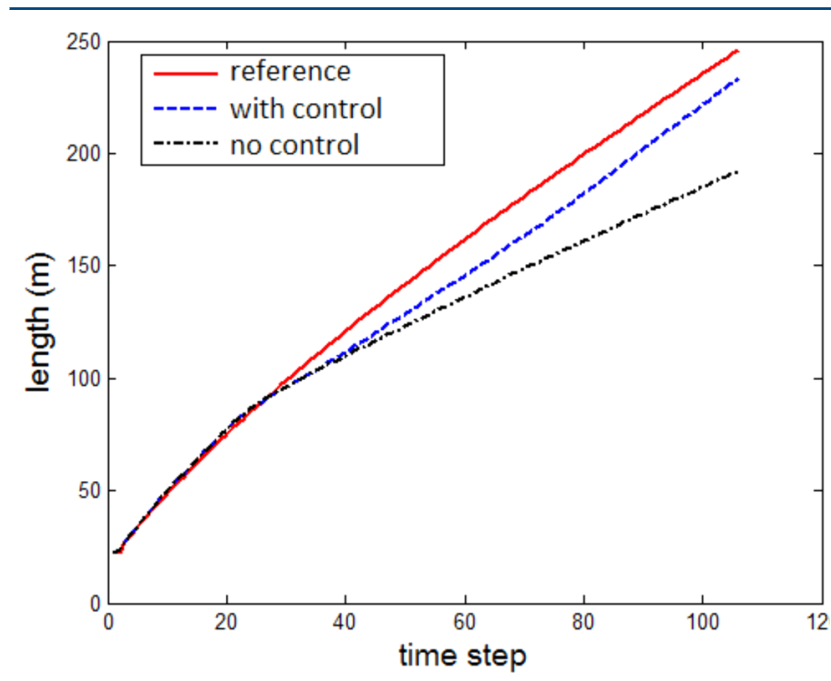

Figure 3. Fracture length change with time. 
The penalty for each variable is given a value of $\lambda=10$. No attempt was made to tune the weights in matrices $\mathbf{Q}$ and $\mathbf{R}$. The movement suppression penalties are decreased by a factor of 10 to better cancel the errors in the state trajectories, but in the limited cases where this was attempted, this adjustment led to oscillatory behavior in both the controller output and state trajectories. It is recommended that future studies should examine the sensitivity of the closed-loop behavior to controller parameter changes as well as optimal selection of the controller weights.

The inclusion of a constant disturbance model within the QDMC formulation provides integral action. ${ }^{37}$ However, due to the fact that the set-point for each of the controlled states is continuously changing during the fracturing process, the system does not reach a steady-state so that integral action can drive the errors to zero.

Figure 4 shows the profile of the fracture width at the wellbore. At time step 20, the disturbance decreases the

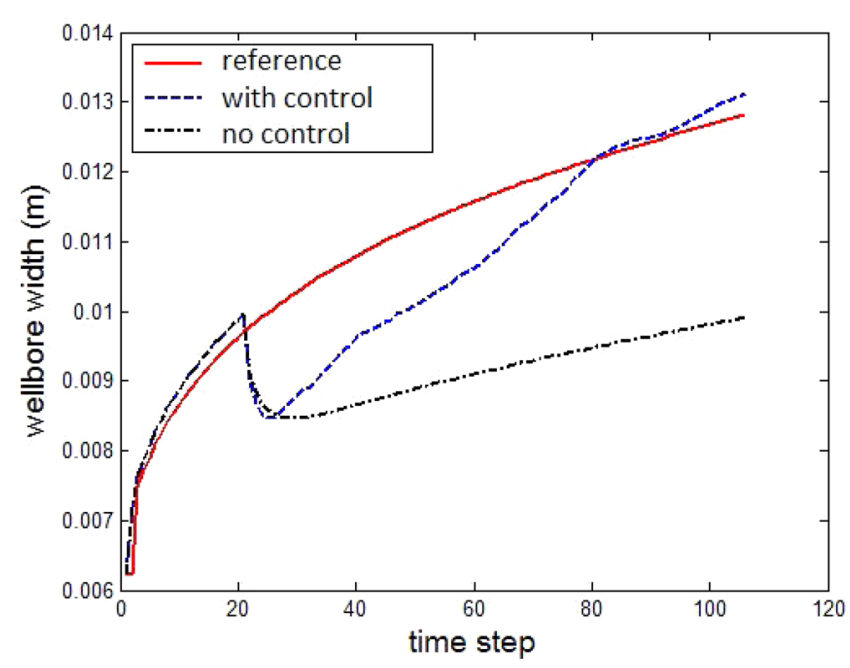

Figure 4. Fracture width at the wellbore change.

injected fluid volume by one-half. At this lower injection rate, because the fracture is still propagating forward and there is fluid leak-off to the formation, the volume of the fluid in the fracture is insufficient to maintain the width, thus contraction occurs. To fully cancel the effect of the disturbance, the injection rate should instantly jump to $0.04 \mathrm{~m}^{3} / \mathrm{s}$; however, it is evident from Figure 4 that the closed-loop provides much less aggressive control. The sharp drop in the fracture width at time step 20 also can be predicted from the pressure-width and lubrication equations, respectively. ${ }^{28}$ Specifically, the net pressure is fixed at the fracture tip. Thus, a change in the pressure gradient due to a decrease in the flow rate implies a drop in the net pressure and also a proportional decrease in the width. It is noted that the QDMC controller drives the fracture width at the wellbore back to the target at approximately time step 100. This is in contrast to the case without feedback control, where the error in wellbore width continues to grow.

At time step 80, proppant injection begins. Figure 5 shows the injected proppant concentration for the case with feedback control. Small increases are made in the injected proppant concentration compared to the nominal input of $0.27(\mathrm{v} / \mathrm{v})$. This change may have resulted from the difference in fracture dimensions (width and length) compared to the reference trajectories, but it could have also occurred if inaccurate

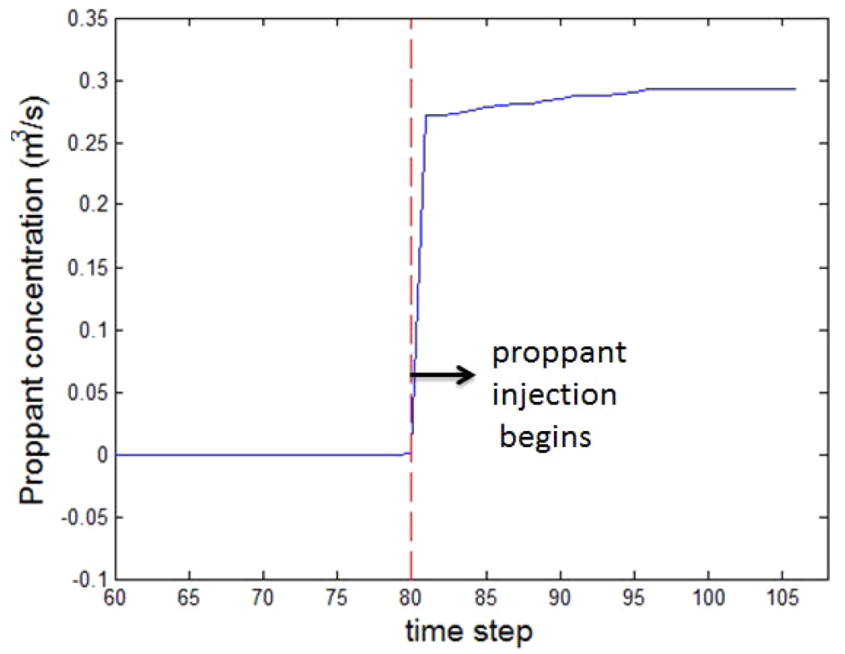

Figure 5. Injected proppant concentration for the case of feedback control.

predictions were provided by the step response model. Figures 6-8 provide results on the fracture geometry and proppant

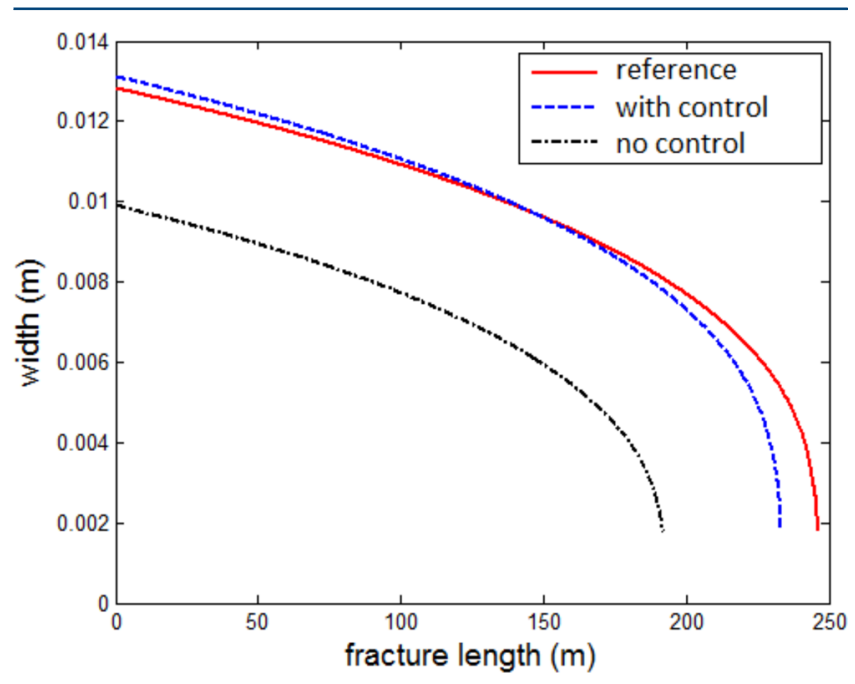

Figure 6. Fracture width at the end of pumping.

distribution after pumping is completed at time step 106. The fracture width profiles across the fracture propagation direction are shown in Figure 6. There exists a much larger offset from the target width in the absence of feedback control versus when control is present. Figure 7 presents the profile of the proppant bank height along the fracture length at the end of pumping. The open-loop results exhibits large errors compared to the closed-loop results. It should be noted that the proppant bank in the closed-loop case has a height distribution whose shape appears to resemble the reference bank.

As previously demonstrated on the nonlinear system model, ${ }^{28}$ the final achieved fracture condition after shut-in is not only dependent on the fracture geometry at the end of pumping, but also dependent on the suspended proppant distribution inside the fracture before the shut-in process begins. The proppant concentration located $5 \mathrm{~m}$ above the fracture bottom was chosen to be controlled. The concentration in this location under open-loop and closed-loop conditions are compared to the reference case in Figure 8. In 


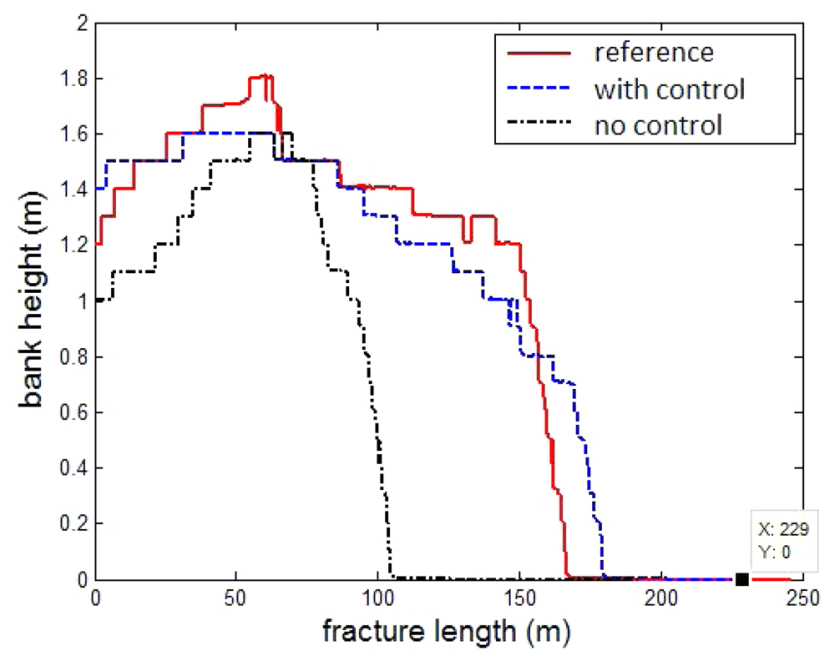

Figure 7. Proppant bank height at the end of pumping.

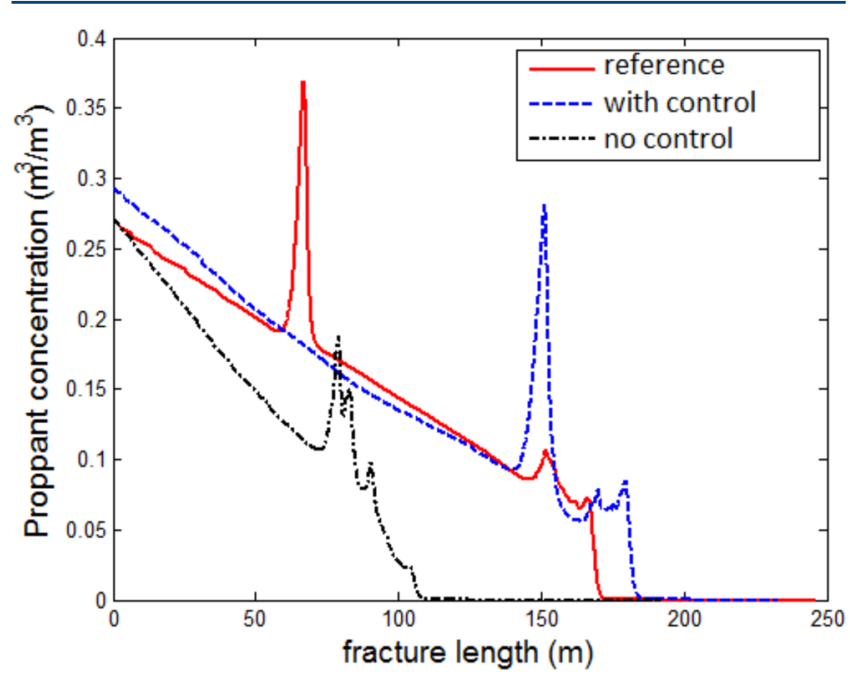

Figure 8. Proppant concentration at the end of pumping, across a band at a height of $5 \mathrm{~m}$.

the former, the flow rate disturbance remained uncorrected, resulting in both less proppant entering the fracture and in the proppant present in the fracture not being transported far into the fracture. In the latter, the proppant concentration profile at the end of pumping shows that the proppant traveled further into the fracture, comparable to the reference case, since the amount of proppant injected increased as a result of the controller's correction of the flow rate.

Pumping progressed to time step 106 (2650 s), then shut-in commenced. During shut-in, it is assumed that all the fluid leaked into the rock formation and that the fracture closed onto the proppant. For each computational cell, if proppant is present in a sufficient amount at the time of closure, the fracture is assumed to be propped at this location, and the associated width is determined; otherwise, the fracture is assumed to have closed completely in this area and no benefit to conductivity is achieved at this location. Figure 9 presents the final propped fracture height after the shut-in process is completed. The height in the closed-loop case shows much smaller deviation from the reference height as compared to the open-loop case.

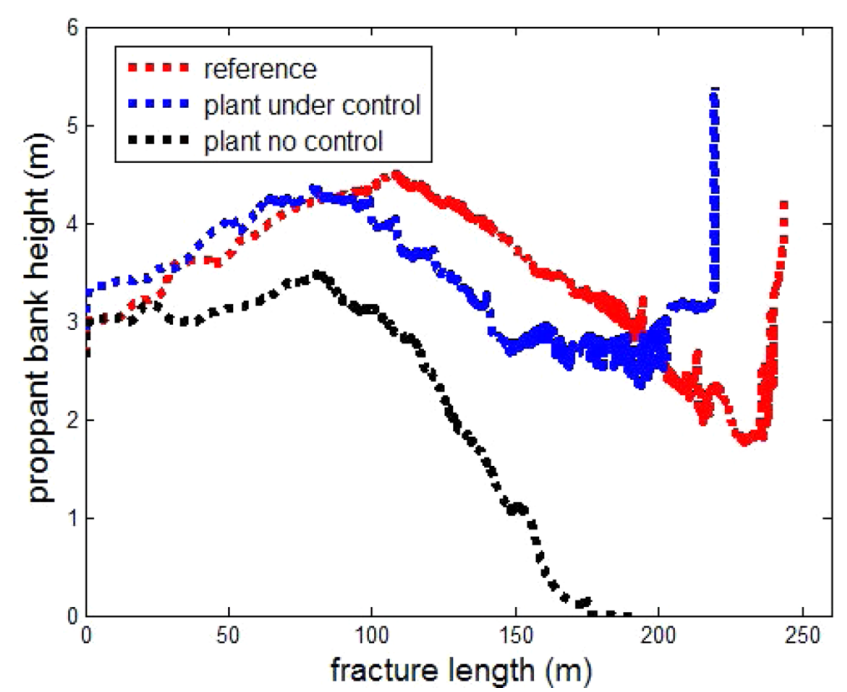

Figure 9. Proppant bank height after the fracture is fully closed.

An important difference between the disturbance responses is that the propped length from closed-loop control is much larger than that from the open-loop case. This advantage can be quantified by the use of an effective fracture length $x_{\mathrm{f}}$ which is defined as the length of the propped fracture where the height is at least $1 \mathrm{~m}$ high (10\% of the open fracture height during pumping). The effective length for open-loop operations $\left(x_{\mathrm{f}}=\right.$ $150 \mathrm{~m})$ is nearly $40 \%$ less than the reference case $\left(x_{\mathrm{f}}=239 \mathrm{~m}\right)$, while in a closed-loop $x_{\mathrm{f}}=235 \mathrm{~m}$ is very close to the target. Figure 10 displays the bounding surfaces of the final fracture achieved under open- and closed-loop operations. It appears that in the presence of the disturbance, both operations show some difference from the target. However, the closed-loop still achieves closer performance to the target.

Several performance criteria important to assess the hydraulic fracturing operations have been computed for both open- and closed- loop operations as listed in Table 2. The propped fracture volume, $V_{\mathrm{p}}$, is the volume of the fracture remaining open after shut-in, which provides a high conductivity path for reservoir depletion. The dimensionless fracture conductivity, $C_{\mathrm{fD}}$, is defined as

$$
C_{\mathrm{fD}}=\frac{\bar{W} K_{\mathrm{f}}}{L_{\mathrm{f}} K_{\mathrm{r}}}
$$

where $K_{\mathrm{f}}$ is the proppant bank permeability, $\bar{W}$ is the average fracture width, $K_{\mathrm{r}}$ is the rock permeability, and $L_{\mathrm{f}}$ is the bank length. Fracture conductivity represents the ratio between the ability of the created proppant bank to conduct oil and gas flow to the ability of the surrounding reservoir to transport these resources. The dimensionless proppant number $N_{\text {prop }}$ is the ratio of the propped volume in the payzone to the total payzone volume ${ }^{38}$ (chosen to be $9.6 \times 10^{4} \mathrm{~m}^{3}$ in this example), with both volumes weighted by their associated permeabilities. Finally, $J_{D}$ is the dimensionless productivity index, which can be determined from the fracture conductivity and proppant number. ${ }^{1}$ For a given amount of consumed material (fluid, proppant, and additive), maximizing $J_{\mathrm{D}}$ is a priority for the fracturing operation, since this measure corresponds to predicted well production. ${ }^{39}$ Comparing each of the measures in Table 2 among the three cases (reference, open-, and closedloop control) supports the conclusion that the closed-loop control aids the fracturing process in achieving close to the 
(a)

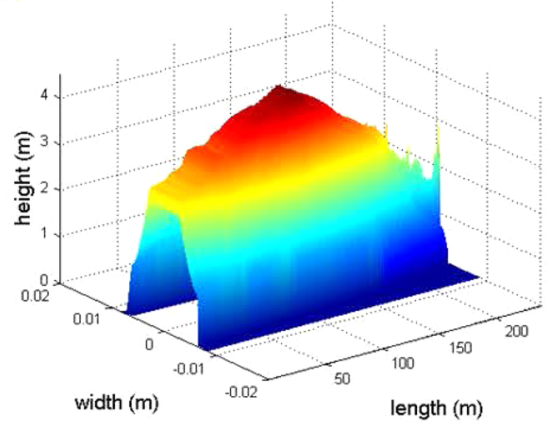

(b)

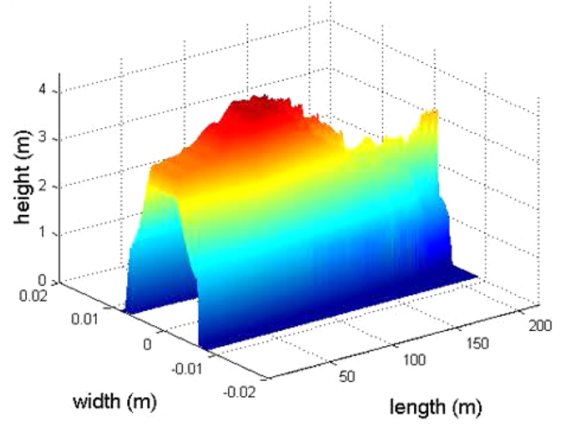

(c)

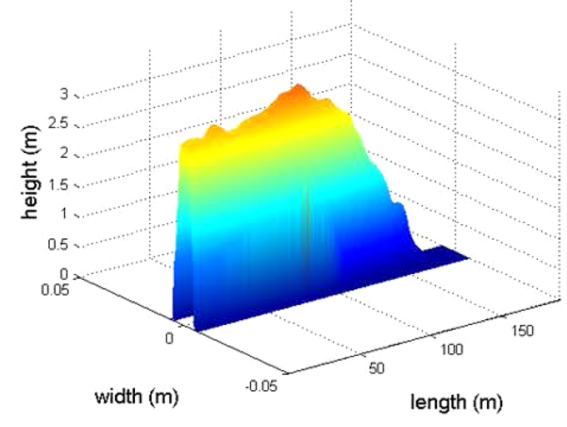

Figure 10. Surface plots of the final propped fracture (a) reference case having no disturbance, (b) with input disturbance and feedback control, and (c) with disturbance but no control.

Table 2. Calculated Performance Criteria for the Hydraulic Fracturing Process after Fracture Close-In

\begin{tabular}{lcrrc}
\multicolumn{1}{c}{ case } & $V_{\mathrm{p}}$ & \multicolumn{1}{c}{$C_{\mathrm{fD}}$} & \multicolumn{1}{c}{$N_{\text {prop }}$} & $J_{\mathrm{D}}$ \\
reference & 6.52 & 9.09 & 6.78 & 1.60 \\
closed-loop & 6.20 & 10.30 & 6.49 & 1.57 \\
open-loop & 2.96 & 8.95 & 13.09 & 1.14 \\
\hline
\end{tabular}

designed performance in the face of an unmeasured disturbance.

For each of the controlled variables, as well as the final fracture condition, there are minor to significant deviations between the reference case and results obtained from the closed-loop system, indicating that the QDMC controller is not completely effective in attenuating the disturbance. However, it should be noted that the study performed herein provides an especially difficult challenge for control since it was assumed that the disturbance was both unmeasured and not modeled within the control structure. Therefore, cancellation of the disturbance relies upon the estimated output bias term of the QDMC controller. Even though the state estimator was not provided with a value of the input disturbance, the particle filter was able to provide acceptable state estimates to the controller based on the available measurements, which consisted only of wellbore width and fracture length.

Better closed-loop performance could be achieved by allowing the particle filter to estimate the input disturbance's magnitude, the system identification module to generate a model containing the disturbance effect, and the QDMC controller to include the identified model and estimated magnitude within predictions of the system trajectory in order to reject the disturbance effects. Nonetheless, though the controller was not particularly configured for the specific disturbance type encountered, the current study demonstrated that the proposed model-based closed-loop system design is advantageous even in this situation.

An ideal combination of model type and MPC algorithm would provide accurate modeling of the system behavior, good controller performance, and low computational requirements for both the model identification and control move calculations. Since the linear step response model is of limited accuracy and its inaccuracy may increase rapidly as the horizon progresses, the use of nonlinear and time-dependent empirical models should be considered for use within the MPC framework. However, as noted previously, it is doubtful that an empirical model can represent this complex system behavior over extended periods of time, thus, particle filtering and repeated system identification also would be required. The use of a nonlinear empirical model in place of the linear step response model may lead to greater validity over a longer horizon before requiring model reidentification. Further investigation is warranted to determine the performance gains of a nonlinear MPC framework.

\section{SUMMARY AND FUTURE WORK}

The current work proposed a framework for closed-loop model-based control of hydraulic fracturing processes. A model introduced within a previous study was used to describe the complex fracture propagation and proppant bank growth phenomena combined with the fluid and solids transport behavior. The control structure relies on particle filtering to estimate the unmeasure states of the system. To achieve modelbased control, frequent system identification is performed on the rigorous but complex computational model in order to generate a simpler step response model for use within a QDMC controller. The proposed control strategy was demonstrated through simulation experiments where the system was subjected to an unknown disturbance. The results indicate that the proposed closed-loop control framework provides a promising approach to achieve feedback control of the fracturing system.

Future work can proceed in several directions. Many physical processes were simplified in this preliminary work including: transport delays from the surface and temperature effects on viscosity. The current model used in the control framework might be improved by considering these effects. Fracture height and leak-off coefficient are two physical parameters that were assumed in this study; however they may have large uncertainties in field applications. The use of particle filtering for state estimation also can allow for simultaneous updating of the estimates of these parameters, but this component has not yet been addressed. Also, the science and technology applied during fracturing treatments are constantly advancing, including the introduction of new materials, such as gelling agents added to the treatment slurry to prevent proppant settling, as well as the use of novel measurement types relying on radiological, optical, or sonic means to provide subterranean sensing information. Subsequent studies on closed-loop control of hydraulic fracturing should incorporate the consequences of these technologies upon the physical description of the system, the fracture treatment design, the job execution, and the final performance, with emphasis on enhancements to the control framework through additional measurement types and manipulated variables. The current work suggests that changes 
to a fracture treatment schedule should not cease before the process begins, instead by "closing the loop" and putting any available information to use in order to perform continuous online adjustment, the benefits of feedback control can be extended within this field of growing importance.

\section{AUTHOR INFORMATION}

\section{Corresponding Author}

*E-mail: karlene.hoo@montana.edu. Phone: 406.994.4145. Fax: 406.994.4733.

\section{Notes}

The authors declare no competing financial interest.

\section{ACKNOWLEDGMENTS}

The first author acknowledges the financial support of the TTU Process Control \& Optimization Consortium.

\section{NOMENCLATURE}

$d=$ proppant diameter, $\mu \mathrm{m}$

$h_{\mathrm{f}}=$ local slurry flow height, $\mathrm{m}$

$q=$ fracturing fluid flow rate, $\mathrm{m}^{3} / \mathrm{s}$

$x=$ lateral fracture propagating direction, $\mathrm{m}$

$t=$ lapse time since start of pumping, $\mathrm{s}$

$z_{k}=$ measurement at time step $k$

$x_{k}=$ state estimate at time step $k$

$x_{k}^{*}=$ target state at time step $k$

$\mathbf{Q}=$ state error weighting matrix

$Q^{0}=$ slurry injection rate

$\mathbf{R}=$ controller movement penalty matrix

$\Delta u_{k}=$ controller movement at time step $k$

$C=$ proppant concentration $(\mathrm{v} / \mathrm{v})$

$C_{1}=$ fluid-loss coefficient, $\mathrm{m} / \sqrt{ } \mathrm{s}$

$C_{\mathrm{fD}}=$ fracture conductivity

$E=$ Young's modulus of the formation

$K_{\mathrm{f}}=$ proppant permeability

$K_{\mathrm{r}}=$ rock permeability

$L=$ fracture length, $\mathrm{m}$

$H=$ fracture height, $\mathrm{m}$

$P=$ net pressure, $\mathrm{MPa}$

$U=$ fluid leak-off velocity, $\mathrm{m} / \mathrm{s}$

$V_{\mathrm{s}}=$ proppant settling velocity, $\mathrm{m} / \mathrm{s}$

$W=$ fracture width, $\mathrm{m}$

$\bar{W}=$ average fracture width, $\mathrm{m}$

$\mu=$ fluid viscosity, $\mathrm{Pa} \cdot \mathrm{s}$

$\nu=$ Poisson ratio of the formation

$\tau(x)=$ fracture opening time at position $x, \mathrm{~s}$

$\phi=$ proppant bank porosity

\section{REFERENCES}

(1) Economides, M., Martin, T., Eds. Modern fracturing: Enhancing natural gas production; Energy Tribune Publishing: TX, USA, 2007.

(2) Economides, M., Nolte, K., Eds. Reservoir stimulation, 3rd ed.; John Wiley \& Sons: NY, USA, 2000.

(3) Gidley, J., Holditch, S., Nierode, D., RW, V., Eds. Recent advances in hydraulic fracturing; Monograph Series; Society of Petroleum Engineers: TX, USA, 1989; Vol. 12; pp 210-222.

(4) Julier, S.; Uhlmann, J. New extension of the Kalman filter to nonlinear systems. Proceedings of SPIE 3068, Signal Processing, Sensor Fusion, and Target Recognition VI, Orlando, Florida, April 21, 1997.

(5) Evensen, G. Journal of Geophysical Research: Oceans 1994, 99, 10143-10162.

(6) Margulis, S.; McLaughlin, D.; Entekhabi, D.; Dunne, S. Water Resour. Res. 2002, 38, 35-1-35-18.
(7) Chen, Y.; Hoo, K. Control Engineering Practice 2012, 20, 10421057.

(8) Chen, Y.; Hoo, K. Systems Science \& Control Engineering: An Open Access Journal 2013, 1, 12-19.

(9) Haugen, V.; Evensen, G. Ocean Dynamics 2002, 52, 133-151.

(10) Gillijns, S.; Mendoza, O.; Chandrasekar, J.; De Moor, B.; Bernstein, D.; Ridley, A. What is the ensemble Kalman filter and how well does it work? Proceedings of the American Automatic Control Conference, Minneapolis, MN, June 14-16, 2006.

(11) Weerts, A. H.; El Serafy, G. Y. Water Resources Research 2006, DOI: $10.1029 / 2005$ WR004093.

(12) Arulampalam, M.; Maskell, S.; Gordon, N.; Clapp, T. IEEE Transactions on Signal Processing 2002, 50, 174-188.

(13) Gay, D.; Ray, W. Chem. Eng. Sci. 1995, 50, 1519-1539.

(14) Chiu, T.; Christofides, P. AIChE J. 1999, 45, 1279-1297.

(15) Christofides, P. Chem. Eng. Sci. 1998, 53, 2949-2965.

(16) Christofides, P.; Daoutidis, P. Finite-dimensional control of parabolic PDE systems using approximate inertial manifolds. Decision and Control, Proceedings of the 36th IEEE Conference, San Diego, CA, Dec 12, 1997; pp 1068-1073.

(17) Christofides, P.; Daoutidis, P. Journal of Process Control 1997, 7, 313-328.

(18) Zheng, D.; Hoo, K.; Piovoso, M. Ind. Eng. Chem. Res. 2002, 41, $1545-1556$

(19) Zheng, D.; Hoo, K. Comput. Chem. Eng. 2004, 28, 1361-1375.

(20) Mahadevan, N.; Hoo, K. Chem. Eng. Sci. 2000, 55, 4271-4290.

(21) Balas, M. Journal of Mathematical Analysis and Applications 1983, 91, 527-546.

(22) Ray, W. Advanced process control; McGraw-Hill: NY, USA, 1981.

(23) Gauthier, J.; Xu, C. International Journal of Control 1991, 53, 45-79.

(24) Hanczyc, E.; Palazoglu, A. Industrial \& engineering chemistry research 1995, 34, 557-566.

(25) Armaou, A.; Christofides, P. Applied Mathematics and Computer Science 2001, 11, 287-318.

(26) Armaou, A.; Christofides, P. Automatica 2001, 37, 61-69.

(27) Baker, J.; Christofides, P. Ind. Eng. Chem. Res. 1999, 38, 43724380 .

(28) Gu, Q.; Hoo, K. Ind. Eng. Chem. Res. 2014, 53, 10491-10503.

(29) Carter, R. In Drilling and Production Practice; Howard, G., Fast,

C., Eds.; American Petroleum Institute: NY, USA, 1957; pp 261-269.

(30) Detournay, E.; Cheng, A.-D.; McLennan, J. J. Energy Resour. Technol. 1990, 112, 224-230.

(31) Nordgren, R. Old Society of Petroleum Engineers Journal 1972, 12, 306-314.

(32) Sneddon, I. Proceedings of the Royal Society of London. Series A. Mathematical and Physical Sciences 1946, 187, 229-260.

(33) Quirein, J.; Grable, J.; Cornish, B.; Stamm, R.; Perkins, T. Microseismic fracture monitoring. SPWLA 47th Annual Logging Symposium, Veracruz, Mexico, June 4-7, 2006.

(34) Chen, T.; Morris, J.; Martin, E. Journal of Process Control 2005, $15,665-673$.

(35) Carpenter, J.; Clifford, P.; Fearnhead, P. IEE Proceedings-Radar, Sonar and Navigation 1999, 146, 2-7.

(36) Zafiriou, E. On the closed-loop stability of constrained QDMC. Proceedings of the American Automatic Control Conference, Boston, MA, June 26-28, 1991; pp 2367-2372.

(37) Maciejowski, J. Predictive control: with constraints; Pearson education: Prentice Hall: NJ, USA, 2001.

(38) Romero, D.; Valko, P.; Economides, M. SPE Production \&Facilities 2003, 18, 57-64.

(39) Diyashev, I.; Economides, M. SPE Production \& Operations 2006, 21, 394-401. 